\title{
The Prevalence of Some Intestinal Parasites in Stray Dogs From Tetova, Fyr Macedonia
}

\author{
Abdilazis Llokmani (Msc), \\ Regional Unit of Food and Veterinary Inspection, FYR Macedonia \\ Dhimitër Rapti (Prof. Dr) \\ Department of Clinical Subjects, Faculty of Veterinary Medicine, \\ Agricultural University of Tirana, Albania
}

doi: 10.19044/esj.2017.v13n21p21 URL:http://dx.doi.org/10.19044/esj.2017.v13n21p21

\begin{abstract}
Stray dogs are a reservoir of intestinal parasites and a source of infection for pet dog owners. Some parasites, including hookworms and roundworms, are zoonotic parasites. This means they can easily be transmitted from pets to humans. The present study aims to determine the prevalence of intestinal parasitic infections in stray dogs located in Tetova, FYR Macedonia region between February to April 2017. A total of 60 samples were examined by centrifugation-flotation methods. The overall prevalence of gastrointestinal parasites were $0,40 \%, 0,13 \%, 0,08 \%$, and 0,01\% for Ancylostoma spp., Trichuris vulpis, Toxocara canis and Coccidia respectively. Stray dogs are associated with a high risk of infection. Also, the periodic laboratory tests can confirm this. The information received from this present study may be useful in the designing of control programs. It was also used in educating the dogs' owners about public health issues of dog keeping, hence reducing the risks associated with zoonotic infections.
\end{abstract}

Keywords: Ancylostoma, Trichuris vulpis, Toxocara canis, Coccidia

\section{Introduction}

The presence of intestinal parasites such as hookworms, roundworms, and whipworms is one of the most frequent problems in dogs. Dogs can acquire intestinal parasites through ingesting parasite eggs or spores in contaminated soil, sand, water, faeces or food. Puppies can also contract these worms from their mother while in utero or during nursing. Intestinal parasitic infections in dogs are commonly recognized as a cause of gastrointestinal disorders with a high prevalence in developing countries around the world (Daryani, 2009). Among intestinal helminthes of dogs, 
Toxocara canis represents the major concern because it can cause severe infection in humans (Katagiri, 2008). Environmental faecal contamination by infected dogs is more or less a source of infection for humans. In fact, parasitic elements like eggs, larvae, cysts, and oocytes excreted via canine faecal route can survive over a long time and be infective in the environment at different conditions. Most of the time, the presence of intestinal parasites do not show symptoms until the infection has become severe. This is why it is important to collect stool samples from dogs periodically and to check them for any parasitic infection. The feacal examination is highly recommended when clinical signs like slowdown of growth, diarrhoea with mucus or blood, swollen abdomen, weight loss, and vomiting are present. The purpose of this study is to determine the prevalence of intestinal parasites in stray dogs in Tetova FYR Macedonia. Samples were taken randomly from 60 stray dogs and the parasite prevalence was calculated.

\section{Materials and Methods}

\section{Sample Collection and Processing}

Faecal samples were collected from stray dogs of different ages between February to April 2017. These samples were collected with the necessary biosafety measures and placed in bottles labeled with numbers for subsequent microscopic diagnosis using the centrifugation-flotation technique. A minimum of 2-6 g of faeces were collected from each animal, and was immediately placed into a plastic container. After then, it was stored at $4^{\circ} \mathrm{C}$ until they were examined.

The feaces samples were mixed thoroughly with $15 \mathrm{~mL}$ pre-made zinc sulphate solution (ZnSO4, specific gravity 1.18) and transferred to a 15$\mathrm{mL}$ conical tube. Additional $\mathrm{ZnSO} 4$ was added to bring the volume up to 15 $\mathrm{mL}$ if required, and the solution was centrifuged for 5 minutes. Following centrifugation, $\mathrm{ZnSO} 4$ was added to form a positive meniscus, onto which a cover slip was placed and left for 5 to 10 minutes. The cover slip was removed, placed on a glass slide, and was examined by light microscopy. The entire cover slip area was examined using a $10 \times$ and $40 \times$ objective.

All dogs that had at least one fecal parasite egg were considered positive. Faecal samples were recorded as positive if $1 \mathrm{egg} / \mathrm{cyst} /$ oocyte was observed in the faecal analysis. The prevalence of infection for each parasite was calculated as the number of positive samples divided by the total number of samples tested and expressed as a percentage.

\section{Results and Discussion}

Stray dogs (Canis familiaris) are ownerless native dogs that roam freely without human supervision. The high prevalence of enteric parasites in stray dogs comes as a result of their feeding on rubbish bins and lack of 
health management. It is also related to high density of stray dogs in some regions, as the one which is in this study. Since dogs live in close proximity with human beings, the zoonotic diseases can be transmitted to humans and cause serious consequences. The transmission of zoonotic parasites could be through indirect contact with dogs secretions and excretions, infected water and food, and through direct contact with the dogs (Lappin, 2002). The most common zoonotic helminth parasites of dogs are Strongyloides stercorslis, $A$. caninum, Dipylidium caninum, T. canis, Echinococcus granulosus, and Trichuris vulpis.

However, three of the above named parasites were found in tested samples in the current study. T.canis and A.caninum are of special concern to veterinarians due to its zoonotic potential. This study documented a high prevalence of intestinal parasitic infections in dogs from Tetova, FYR Macedonia. A total of 60 faecal samples were analyzed within 3 months (February to April 2017). The intestinal parasites found in these dogs were Ancylostoma spp. (0.40\%); Trichuris vulpis $(0.13 \%) ; \quad$ Toxocara canis (0.08\%); and Coccidia (0.01\%).

Table 1. Number of Infected Feaces and Parasite Found

\begin{tabular}{|c|c|}
\hline Parasite & No. of Infected Feaces/60 \\
\hline Ancylostoma spp. & 24 \\
\hline Trichuris vulpis & 8 \\
\hline Toxocara canis & 5 \\
\hline Coccidia & 1 \\
\hline Negative samples & 26 \\
\hline
\end{tabular}

During this study, the concurrent infection with two or more parasite species was very common. It was observed that the young dogs' faeces showed a higher number of positive samples. Thus, the age of dogs is significant when it comes to parasites burden. Young animals were found to more frequently shed parasites eggs in faeces than adult animals (Gates, 2014). No effect of the season in the occurrence of the different parasite genera was observed, except for Ancylostoma spp. Therefore, an increase in the percentage of dogs shedding eggs was observed during April. This parasite was observed in $0.40 \%$ of samples. The overall frequency of $T$. canis obtained from tested samples was $0.08 \%$. It is important to mention that these worms are very resistant to environmental changes (Jordan, 1993). Also, they present a high risk for human infection. Trichuris vulpis is on occasion stated to be a zoonotic infection, but the data is not convincing.

Human whipworms (Trichuris trichiura) sometimes produce large eggs that are similar in size to Trichuris vulpis. These abnormal eggs, alongside the regular-sized and smaller Trichuris trichiura eggs in a human fecal, can resemble the eggs of Trichuris vulpis. Veterinarians play a critical 
role in these situations because controlling the intestinal parasites in dogs can be an effective strategy for minimizing the risk of infection in people (Traversa, 2014; Paul, 2010). In this study, the centrifugation technique was used, and was shown to be a much more sensitive test than the simple flotation method (Dryden, 2005). The current study showed a high prevalence of different gastrointestinal parasites of stray dogs from Tetova, FYR Macedonia. Due to regional variations in parasite prevalence, such information is often of limited value outside the specific areas evaluated. The possible reasons for the wide range of differences amongst the gastrointestinal parasites' prevalence might be due to factors like: geographical location, the presence or absence of the intermediate hosts of the corresponding parasites, sampling protocols, demographic factors, anthelmintic usage, and diagnostic techniques (Gugsa, 2015).

\section{Conclusion}

Ancylostoma spp. was the most common parasite detected in stray dogs in this study $(0.40 \%)$. The parasite was observed as single in a mixed infection.

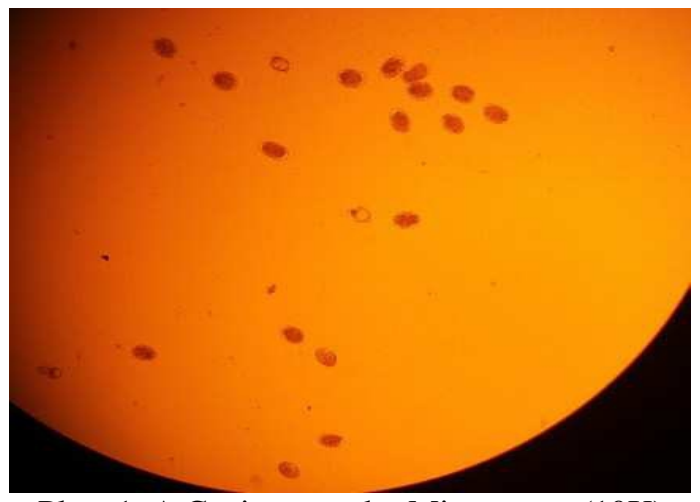

Plate 1. A.Caninum under Microscope (10X)

The data obtained from this study may be very useful in designing an effective prevention and control programs for home kept dogs that go for walks in the same areas with stray dogs. It is also important to provide appropriate public health education to the dogs' owners to reduce the risks of zoonotic infections through the use of anthelmentics. Early diagnosis for the presence and type of intestinal parasite is vital. Also, veterinarians should formulate and prescribe a schedule for deworming the dogs on a regular basis all through the year. This study and others that will follow should be taken into consideration for designing a health management program for pet dogs with a regular and appropriate internal parasite control strategy for stray dogs. 


\section{References:}

1. Daryani A, Sharif M, Amouei A, \& Gholami S (2009). Prevalence of Toxocara canis in stray dogs, northern Iran. Pak J Biol Sci.;12:1031-1035.

2. Dryden MW, Payne PA, \& Ridley R (2005). Comparison of common fecal floatation techniques for the recovery of parasite eggs and oocytes. Vet Ther.; 6:15-28.

3. D Traversa, A Frangipane di Regalbono, A Di Cesare, F La Torre, J Drake, M Pietrobelli (2014). Environmental contamination by canine geohelminths. Parasit Vectors, 7, p. 67.

4. Gates MC, \& Nolan TJ (2009). Endoparasite prevalence and recurrence across different age groups of dogs and cats. Vet Parasitol. ;166:153-158.

5. Getachew Gugsa, Tesfay Hailu, Shewit Kalayou, Nigus Abebe \& Yohannes Hagos. Prevalence and Worm Burdens of Gastro-Intestinal Parasites in Stray Dogs of Mekelle City, Tigray, Ethiopia, Mekelle University, College of Veterinary Medicine, Mekelle, Ethiopia 1 Shire Agricultural Technical and Vocational and Education Training College, Shire, Ethiopia.

6. Katagiri S, \& Oliveira-Sequeira TC (2008). Prevalence of dog intestinal parasites and risk perception of zoonotic infection by dog owners in Sao Paulo State, Brazil. Zoonoses Public Health.;55:406413.

7. Lappin MR (2002). Pet ownership by immunocompromised people. Bayer Zoonosis Symposium, North American Veterinary Conference, 24(5): 16-25.

8. Jordan, H. E., S. T. Mullins, and M. E. Stebbins, 1993: Endo-

9. parasitism in dogs: 21,583 cases (1981-1990). J. Am. Vet.

10. Med. Assoc. 203, 547-549.

11. Jordan, H. E., S. T. Mullins, and M. E. Stebbins, 1993: Endo-

12. parasitism in dogs: 21,583 cases (1981-1990). J. Am. Vet.

13. Med. Assoc. 203, 547-549.

14. Jordan, H. E., S. T. Mullins, and M. E. Stebbins (1993). Endoparasitism in dogs: 21,583 cases (1981-1990). J. Am. Vet.Med. Assoc. 203, 547-549.

15. M Paul, L King, \& EP Carlin (2010). Zoonoses of people and their pets: a US perspective on significant pet-associated parasitic diseases. Trends Parasitol, 26, pp. 153-154.

16. PR Torgerson, \& CN Macpherson (2011). The socioeconomic burden of parasitic zoonoses: global trends. Vet Parasitol, 182, pp. 79-95. 